\title{
Update on postoperative pain management
}

There is currently an unusual degree of recognition amongst anaesthetists and the newly emerging acute pain therapists that older techniques of acute postoperative pain control are obsolete and for the most part inadequate in treating acute pain. ${ }^{1}$ Much of the success of the recent drive to correct this situation has been derived from innovation and new information relating to techniques of administration of opioids, local anaesthetic agents and other analgesics rather than the development of new agents. This presentation will review the current status of the most effective means of postoperative pain control in current use.

\section{Opioid administration}

Opioids are still the most commonly utilized analgesic for postoperative pain. Routes of administration include oral mucosal, nasal mucosal, rectal mucosal, timed oral release preparations, transdermal, intramuscular, intravenous (continuous infusions, PCA), and spinal (epidural, intrathecal).

\section{Intravenous opioids: pulse dose infusions}

Continuous intravenous infusion of opioid is a relatively simple technique and is preferable to both $i v$ bolus dosing and $\mathrm{im} / \mathrm{sc}$ administration in those situations involving the early postoperative period or in the ICU. ${ }^{2}$ Due to the monitoring requirements of continuous opioid infusions when used as the sole analgesic regimen, an intensive or acute care-setting is required. Opioids used systemically must be front-loaded to achieve the minimum effective concentration (MEAC) in a reasonable time period. The use of an intravenous opioid infusion without front-loading will not result in the achievement of MEAC for at least three elimination half-lives. Stevens ${ }^{3}$ has summarized guidelines for the estimation of front-loading various opioids as well as simple methods of calculating infusion rates. A simpler technique involves intravenous loading with methadone which provides prolonged analgesia due to the drug's very long terminal half-life (25-52 hr). Postoperative patients given methadone as a bolus often had extremely prolonged analgesia with only $35 \%$ requiring additional opioids after $18 \mathrm{hr} .{ }^{4,5}$

\section{Patient-controlled analgesia}

Patient-controlled analgesia (PCA) implies the delivery of opioids and other analgesics by any route of administration (e.g., oral, $i v, s c$, epidural) upon immediate patient demand in adequate quantities. ${ }^{6}$ In general, opioid PCA via electronic computer-controlled or mechanical pumps using intermittent iv bolus with or without a low-dose background infusion is the commonest technique used. This system has gained widespread acceptance because of its demonstrated advantages. These include improved analgesia which caters for the inherent inter-patient variability (up to five-fold difference) in requirements and rapid treatment of incident pain. In addition, early simple trials point to improved pulmonary function, decreased respiratory complications, earlier ambulation and reduced hospital stay ${ }^{7,8}$ Disadvantages include high capital costs, mechanical problems (rare), user error - either the programmer (doctor, nurse, pharmacist etc.) or the patient misusing the devices and a very small number of reported overdoses. ${ }^{9}$ Respiratory depression was virtually absent in PCA patients in a recent study comparing PCA morphine to transdermal fentanyl postoperatively. ${ }^{10}$

\section{Spinal opioids}

The use of spinal (epidural, intrathecal) opioids has grown rapidly since their first aplication in 1979. Besides postoperative analgesia, spinal opioids are used for labour analgesia, cancer pain, trauma analgesia, and some chronic benign pain conditions. Delivery methods include intermittent bolus injection with or without continuous infusion, continuous infusion alone or PCA techniques. Virtually all the known opioids in clinical practice have been used including agonist/antagonist compounds. Morphine is the most widely used opioid spinally and is typical of lipidinsoluble opioids. Its epidural administration requires smaller doses (3-7 mg) than when given systemically although there is considerable absorbtion via the epidural veins early after administration." Catheter placement along the vertebral column does not affect the production of analgesia with morphine, ${ }^{12}$ although onset of analgesia can be prolonged in segments some distance from the catheter site. Subarachnoid injection requires very small doses $(0.2-0.5 \mathrm{mg})$ with minimal systemic absorption and provides prolonged analgesia, as does the epidural route

From the Department of Anaesthesia, Toronto General Division, The Toronto Hospital, University of Toronto, Toronto, Ontario. 
(8-24 hr).$^{13}$ Lipophilic opioids, of which fentanyl and sufentanil are typical, seem to act differently. Doses similar to systemic requirements are necessary to achieve analgesia and catheter placement close to the segments where analgesia is desired is important. Systemic uptake is high with lipophilic opioids and, if continuous infusions of fentanyl are used, plasma concentrations may be identical to that found when the drug is given intravenously to produce similar degrees of analgesia. ${ }^{14,15}$ However, if the catheter is placed close to the site of surgery, smaller doses of fentanyl are required with subsequent lower plasma concentrations. ${ }^{16}$ The increased dosage requirements with lipophilic opioids may be related to increased systemic uptake as well as to non-specific binding in the spinal cord. ${ }^{17}$ Side effects of spinal opioids are similar to those seen with alternative delivery routes and include respiratory depression, urinary retention, pruritus, and nausea and vomiting. Respiratory depression after spinal opioids has been studied in far greater detail than with any other delivery route. ${ }^{18}$ Associated risk factors including advanced age, impaired respiratory function, and concomitant administration of parenteral opioids and sedatives. Early respiratory depression results from systemic uptake and redistribution to the respiratory centre and occurs up to 60 min after injection. ${ }^{18}$ Late respiratory depression is related to CSF movement in the neuraxis ${ }^{19}$ and occurs several (up to 18) hours after administration. ${ }^{18}$ With morphine, the risks of respiratory depression are small but may be unpredictable. Fentanyl seems to have less risk of respiratory depression ${ }^{18}$ although with increasing doses it becomes apparent, especially with epidural infusions. ${ }^{20}$ Respiratory depression can be treated with naloxone, whilst the other side effects can be treated with agents specific to the symptoms (diphenhydramine, anti-emetics) or naloxone. There is no consensus as to where and how to monitor patients receiving spinal opioids although somnolence plus respiratory rate may be useful in detecting respiratory depression. In addition, the use of smaller, more frequent doses of morphine may decrease the risk of respiratory depression. ${ }^{21}$ Outcome studies are now appearing indicating that spinal opioids may have distinct benefits in terms of hospital stay, morbidity and mortality. ${ }^{22.23}$

\section{Spinal opioids plus local anaesthetics}

Attempts to decrease the dose and limit the absorption of opioids has led to the use of mixtures of local anaesthetic and epidural opioids. Morphine $\left(0.1 \mathrm{mg} \cdot \mathrm{ml}^{-1}\right)$ plus bupivicaine $0.1 \%$ has been shown to be an effective mixture although no better than epidural morphine alone in terms of analgesia, total opioid requirements or incidence of side effects. ${ }^{24}$ Similarly, epidural fentanyl infusions $\left(10 \mu \mathrm{g} \cdot \mathrm{ml}^{-1}\right)$, or sufentanil $\left(1 \mu \mathrm{g} \cdot \mathrm{ml}^{-1}\right)$ plus bupivicaine $0.1 \%$ have been shown to be effective for postoperative analgesia after various procedures. ${ }^{24} \mathrm{How}$ ever, when similar combinations were subjected to rigorous examination in randomized, double-blind trials, no synergestic effects of the combination were seen compared with the opioid alone. ${ }^{24}$ Mixtures with higher concentrations of bupivicaine $(0.5 \%)$ have produced profound analgesia but patients also develop a high thoracic sensory level. ${ }^{25}$

\section{Transdermal opioids}

The transdermal route has been developed for fentanyl and has had extensive study in relation to acute pain control. ${ }^{10,26}$ The development of the transdermal therapeutic system (TTS) and its application incorporating fentanyl provides a simple continuous non-invasive infusion device with a surprisingly stable therapeutic plasma concentration. ${ }^{26}$ Unfortunately, the system requires $12-18 \mathrm{hr}$ after application to reach the therapeutic concentration with a similar prolonged plasma concentration after removal. Thus the device can produce prolonged stable, plasma levels for up to $72 \mathrm{hr}$. The dosage range available is 25,50 , 75 and $100 \mu \mathrm{g} \cdot \mathrm{hr}^{-1}$. TTS-fentanyl can provide complete analgesia for a small number of patients after surgery but, in general, requires supplementation with either $i v$ or im opioids in small doses. ${ }^{10,27}$ The incidence of nausea and vomiting has been high in some studies. ${ }^{10}$ In addition, some degree of respiratory depression occurs in most of the published studies ${ }^{10}$ especially if larger TTS-fentanyl patches are used. Advantages of the TTS-fentanyl patch include ease of use, non-labour intensive, high patient acceptability, and effective baseline analgesia with minimal to moderate opioid supplementation. Disadvantages include non-titratable, preselected dose, slow onset and preoperative patch application, supplemental analgesic requirement especially in the PACU, and similar requirements for postoperative nursing observation as with any effective opioid analgesic regimen.

\section{Alternative spinal agents}

Local anaesthetic agents have long been used epidurally for postoperative analgesia. Unlike spinal opioids, spinal local anaesthetics, as well as producing analgesia by axonal blockade, are able to decrease the endocrinemetabolic response to surgery, reduce neutropenia and granulocytosis, improve nitrogen balance $e^{28}$ and may reduce the risk of thromboembolic events. ${ }^{29}$ In addition, reduction in pulmonary dysfunction after abdominal and thoracic surgery ${ }^{28}$ and rapid recovery of intestinal motility have been demonstrated. ${ }^{30}$ The disadvantages of epidural local analgesia include motor blockade, sympathetic blockade, systemic local anaesthetic toxicity and urinary retention all of which can be minimized by placing the 
catheter close to the site of surgery. Bupivicaine, the most commonly used agent, is unlikely to be toxic at infusion rates below $30 \mathrm{mg} \cdot \mathrm{hr}^{-1}$. Recent interest in the use of 28 gauge spinal catheters and continuous local anaesthetic infusions for postoperative analgesia ${ }^{31}$ has been tempered by reports of patients developing cauda equina syndromes associated with the spinal catheters and the local anaesthetics used. ${ }^{32}$ Clonidine, an alpha- 2 receptor agonist, has had only minimal success in producing analgesia when administered epidurally. ${ }^{33}$

\section{Peripheral blocks}

Transmission along sensory nerves can be blocked by a variety of techniques using local anaesthetics or by the use of a cryoanalgesia probe. Effective local anesthetic block of peripheral nerves to produce postoperative analgesia can be achieved with wound infiltration, peripheral nerve, intercostal, interpleural and major plexus blocks. ${ }^{34}$ Interpleural analgesia produces continuous, partial block of several intercostal nerves and seems to be very effective for post-cholecystectomy pain ${ }^{35}$ but less effective postthoracotomy. ${ }^{36}$ Clear benefits from this technique have not been shown yet. ${ }^{37}$ Complications include pneumothorax, pleural effusion and systemic local anaesthetic toxicity as well as mechanical problems. Intermittent procedures (intercostal blocks etc.) are very effective but suffer from the drawback of repeat administration for long-term use. For this reason, continuous peripheral blocks have attracted attention and have been used successfully for postoperative analgesia. Continuous techniques have been developed for intercostal catheters (placed at the time of thoracotomy), brachial plexus catheters, lumbar plexus catheters as well as subcostal or paravertebral catheters. ${ }^{34}$

Cryoanalgesia involves the rapid freezing of exposed nerves at thoracotomy and can provide prolonged postoperative analgesia with little complication. ${ }^{38}$

\section{Non-opioid analgesic agents}

The use of anti-inflammatory agents in the postoperative period is increasing as studies indicate injectable agents such as ketorolac have useful analgesic properties especially for mild to moderate pain. ${ }^{39}$ For severe pain these agents may have a pronounced opioid sparing effect. Other useful non-steroidal anti-inflammatory agents include piroxacam, diclofenac and indomethacin, ${ }^{40}$ all of which are administered orally or rectally. Side effects are primarily related to the gastrointestinal tract or platelet inhibition and potential bleeding

\section{Conclusion}

Numerous postoperative analgesic therapies are continuing to develop as interest increases in the control of acute pain, particularly within the specialty of anaesthesia. Further progress will be made in the near future in relation to preemptive analgesia and reduction of postoperative pain by controlling spinal cord plasticity. ${ }^{41,42}$ The concept of multimodal or balanced analgesia ${ }^{43}$ in which the combined use of specific agents blocking specific segments of the pain pathway is another area which may provide improvements in postoperative analgesia.

\section{References}

1 Oden RV. Acute postoperative pain: incidence, severity, and the etiology of inadequate treatment. Anesthesiology Clinics of North America 1989; 7: 1, 1-15.

2 Edwards WT, Breed RJ. The treatment of acute postoperative pain in the postanesthesia care unit. Anesthesiology Clinics of North America 1990; 8: 2, 235-65.

3 Stevens DS, Edwards WTE. Management of pain in intensive care setting. In Press, Anesthesiology Clinics of North America, June 1992.

4 Gourlay GK, Willis RJ, Wilson PR. Postoperative pain control with methadone: influence of supplementary methadone doses and blood-concentration-response relationships. Anesthesiology 1984; 61: 19-26.

5 Gourlay GK, Wilson PR, Glynn CJ. Pharmacodynamics and pharmacokinetics of methadone during the perioperative period. Anesthesiology 1982; 57: 458-67.

6 Ferrante FM. Patient controlled analgesia. (In press), Anesthesiology Clinics of North America, June 1992.

7 Ready B. Patient-controlled analgesia - does it provide more than comfort? Can J Anaesth 1990; 37: 719-20.

8 Wasylak TJ, Abbott FV, English MJM, Jean ME. Reduction of postoperative morbidity following patientcontrolled morphine. Can J Anaesth 1990; 37: 726-31.

9 Thomas $D W$, Owen $H$. Patient controlled analgesia: the need for caution. Anesthesia 1988; 43: 770-2.

10 Sandler AN. Transdermal fentanyl - acute analgesic clinical studies. (In Press), Journal of Pain Management Symposium, 1992.

11 Gustaffson LL, Friberg-Nielsen S, Garle M, et al. Extradural and parenteral morphine: kinetics and effects in postoperative pain. A controlled clinical study. Br J Anaesth 1982; 54: 1167-74.

12 Fromme GA, Steidl $W$, Danielson DR. Comparison of lumbar and thoracic epidural morphine for relief of postthoracotomy pain. Anesth Analg 1985; 64: 454-5.

13 Jacobson L, Chabal C, Brody MC, Ward RJ, Ireton RC. Intrathecal methadone and morphine for postoperative analgesia: a comparison of the efficacy, duration and side effects. Anesthesiology 1989; 70: 742-6.

14 Panos L, Sandler AN, Stringer DG, et al. Epidural vs intravenous fentanyl infusions in postthoracotomy patients: analgesic and pharmacokinetic effects. Anesthesiology, 1990; 73: A831.

15 Loper KA, Ready LB, Downey M, et al. Epidural and 
intravenous fentanyl infusions are clinically equivalent following knee surgery. Anesth Analg, 1990; 70: 72-5.

16 Salomaki TE, Laitinen JO, Nuutinen LS. A randomized double-blind comparison of epidural versus intravenous fentanyl infusion for analgesia after thoracotomy. Anesthesiology 1991; 75: 790-5.

17 McQuay HJ, Sullivan AF, Smallman K, Dickenson AH. Intrathecal opioids, potency and lipophilicity. Pain 1989; 36: 111-5.

18 Etches $R$, Sandler AN, Daley MD. Respiratory depression and spinal opioids. Can J Anaesth 1989; 36: 165-85.

19 Nordberg $C$. Pharmacokinetic aspects of spinal morphine analgesia. Acta Anaesthesiol Scand 1984; 28: Suppl 79: 1-38.

20 Badner NH, Sandler AN, Koren G, Lawson SL, Klein J, Einarson $T R$. Lumbar epidural fentanyl infusions for post-thoractomy patients: analgesic, respiratory, and pharmacokinetic effects. Journal of Cardiothoracic Anesthesia 1990; 4: 543-51.

21 Ready $L B$, Loper KA, Nessly $M$, Wild L. Postoperative epidural morphine is safe on surgical wards. Anesthesiology $1991 ; 75:$ 452-6.

22 Rawal N, Sjostrand U, Christoffersson E, Dahlstrom B, Arvill A, Rydman H. Comparison of intramuscular and epidural morphine for postoperative analgesia in the grossly obese: influence on postoperative ambulation and pulmonary function. Anesth Analg 1984; 63: 583-92.

23 Yeager MP, Glass DD, Neff RK, Brink-Johnsen T. Epidural anesthesia and analgesia in high-risk surgical patients. Anesthesiology 1987; 66: 729-36.

24 Badner N. Epidural agents for post-operative analgesia. (In Press), Anesthesiology Clinics of North America, June 1992.

25 Lee A, Simpson D, Whitfield A, Scott DB. Postoperative analgesia by continuous extradural infusion of bupivicaine and diamorphine. Br J Anaesth 1988; 60: 845-50.

26 Hill $H F$. Clinical pharmacology of transdermal fentanyl. Eur J Pain 1990; 11: 81-91.

27 McLesky $\mathrm{CH}$. Fentanyl TTS for postoperative analgesia. Eur J Pain 1990; 11: 92-7.

28 Torda TA. Management of acute and postoperative pain. International Anesthesiology Clinics 1983; 21: 27-46.

29 Wildsmith JAW. Developments in local anaesthetic drugs and techniques for pain relief. Br J Anaesth 1989; 63: 159-64.

30 Ahn H, Bronge A, Johansson K, Ygge H, Lindhagen J. Effect of continuous epidural analgesia on intestinal motility. Br J Surg 1988; 75: 1176-8.

31 Bevacqua BK, Slucky AV, Adusumilli $S B$. Postoperative analgesia with continuous intrathecal lidocaine infusion. Regional Anesthesia 1991; 15 (suppl 1): 44.
32 Rigler ML, Drasner K, Krejcie TC, et al. Cauda equina syndrome after continuous spinal anesthetics. Anesth Analg 1991; 72: 275-81.

33 Bonnet F, Boico O, Rostaing S, Loriferne JF, Saada $M$. Clonidine-induced analgesia in postoperative patients: epidural vs intramuscular administration. Anesthesiology 1990; 72: 423-7.

34 Panos L, Panos A. Regional and local block of pain. (In Press), Anesthesiology Clinic of North America, June 1992.

35 Reiestad F, Stromskag KE. Interpleural catheter in the management of postoperative pain - a preliminary report. Regional Anesthesia 1986; 11:89-91.

36 Lee $V C, A b r a m S E$. Intrapleural administration of bupivicaine for post-thoracotomy analgesia. Anesthesiology 1987; 66: 586.

37 Schroeder $D$, Baker $P$. Interpleural catheter analgesia after cholecystectomy: the surgical perspective. Aust NZ J Surg 1990; 60: 689-94.

38 Gough JD, Williams AB, Vaughan RS, Khalil JF, Butchart $E G$. The control of post-thoracotomy pain. A comparative evaluation of thoracic epidural fentanyl infusions and cryoanalgesia. Anaesthesia 1988; 43: 780-3.

39 Stanski $D R$, Cherry $C$, Bradley $R$, et al. Efficacy and safety of single doses of intra muscular ketorolac tromethamine compared with meperidine for postoperative pain. Pharmacotherapy 1990; 10: 40S-45S.

40 Miller LG, Pritchard JG. Current issues in NSAID therapy. Primary Care 1990; 17: 589-601.

41 Kavanagh BP, Katz J, Sandler AN, Nierenberg H, Panos $L$. Pre-incisional versus post-incisional lumbar epidural fentanyl reduces pain following thoracic surgery: a randomized double-blind crossover study. Anesthesiology, 1991; 75: A1087.

42 Bach $S$, Noreng MF, Tjellden NU. Phantom limb pain in amputees during the first 12 months following limb amputation, after preoperative lumbar epidural blockade. Pain 1988; 33: 297-301.

43 Dahl JB, Rosenberg J, Dirkes WE, Mogensin T, Kehlet $H$. Prevention of postoperative pain by balanced analgesia. $\mathrm{Br}$ J Anaesth 1990; 64: 518-20. 


\section{Mise à jour sur le traitement de la douleur postopératoire}

On reconnait maintenant de façon courante chez les anesthésistes et les thérapeutes de la douleur aiguë, que les anciennes techniques de contrôle de la douleur postopératoire sont dépassées et pour la plupart inadéquates pour traiter la douleur aiguë.' Une grande partie des succès récents survenus dans la correction de cette situation provient d'innovations et d'informations nouvelles reliées plutôt aux techniques d'administration d'opiacés, d'anesthésiques locaux et autres analgésiques, qu'au développement de nouveaux agents pharmacologiques. Cette présentation passera en revue les moyens actuels les plus efficaces pour contrôler la douleur postopératoire.

\section{L'administration d'opiacés}

Les opiacés sont encore les analgésiques les plus utilisés pour le contrôle de la douleur postopératoire. Les voies d'administration comprennent les muqueuses orale, nasale et rectale, les préparations à libération lente, les voies transdermique, intramusculaire, intraveineuse (infusion continue, PCA), et rachidienne (épidurale, intrathécale).

\section{Opiacés intraveineux : dose intermittente/infusion}

L'administration intraveineuse continue d'opiacés est une technique relativement simple qui demeure préférable autant au bolus intraveineux qu'à l'administration intramusculaire ou sous-cutanée dans les situations postopératoires ou de soins intensifs. ${ }^{2}$ Les besoins de surveillance des infusions continues d'opiacés lorsqu'utilisées comme seul traitement analgésique, demande une surveillance en soins intensifs ou en unité de soins aigus. Les opiacés administrés par voie systémique exigent une précharge pour l'obtention d'une concentration minimale effective dans une période de temps raisonnable. L'utilisation d'une infusion intraveineuse d'opiacés sans précharge ne produira pas cette concentration efficace minimale pour au moins trois demie-vie d'élimination. Stevens ${ }^{3}$ a résumé les recommandations autant pour l'évaluation des doses de précharge d'opiacés que pour les méthodes simples de calcul du taux d'infusion. Une méthode plus simple implique une charge intraveineuse avec la méthadone qui donne une analgésie prolongée à cause de sa demie-vie d'élimination très longue (25-52 heures). Les patients qui ont reçu un bolus de méthadone en postopératoire bénéfi- cient d'une analgésie extrêmement prolongée et seulement $35 \%$ de ceux-ci ont besoin d'opiacés additionnels après 18 heures. ${ }^{4,5}$

\section{Analgésie auto-contrôlée}

L'analgésie auto-contrôlée «PCA » implique l'administration en quantité adéquate d'opiacés et autres analgésiques par plusieurs voies d'administration (buccale, $i v, s c$, épidurale) en réponse à une demande immédiate du patient. ${ }^{6}$ En général, l'administration d'opiacés par PCA à partir de pompes mécaniques ou à contrôle électronique, par bolus intraveineux intermittents, avec ou sans infusion de base, demeure la technique la plus fréquemment utilisée. Ce système a acquis une popularité importante à cause de ses avantages démontrés. Ceux-ci comprennent une analgésie améliorée qui tient compte de la variabilité inhérente entre les patients dans leurs besoins (différence allant jusqu'à cinq fois) et un traitement rapide de la douleur immédiate. De plus, les premiers essais cliniques simples ont démontré une fonction pulmonaire améliorée, une diminution des complications respiratoires, une mise sur pied rapide et des séjours hospitaliers raccourcis. ${ }^{7,8}$ Les désavantages comprennent les coûts d'implantation, les problèmes mécaniques (rares), les erreurs par les utilisateurs - provenant autant du programmeur (médecins, infirmière, pharmacien, etc.) que des patients qui utilisent mal l'appareillage et aussi un très petit nombre de surdoses mentionnées dans la littérature. ${ }^{9}$ La dépression respiratoire est à peu près absente chez les patients utilisant le PCA dans un travail récent qui compare l'administration de morphine par $\mathrm{PCA}$ à celle du fentanyl par voie transdermique en postopératoire. $^{10}$

\section{Les opiacés administrés par voie rachidienne}

L'utilisation d'opiacés par voie rachidienne (épidurale, intrathécale) s'est accrue rapidement depuis la première utilisation en 1979. Au delà de leur utilisation en postopératoire, les opiacés rachidiens sont utilisés pour la douleur pendant le travail obstétrical, la douleur due au cancer, au traumatisme et à certaines conditions chroniques bénignes. Les méthodes d'administration comprennent l'injection de bolus intermittents avec ou sans infusion continue, l'infusion continue seule ou les techniques par PCA. À peu près tous les opiacés utilisés en pratique clinique ont été utilisés 
incluant les agonistes/antagonistes. La morphine est l'agent le plus fréquemment utilisé par voie rachidienne et a un comportement typique des opiacés peu solubles dans les lipides. Son administration épidurale demande des doses plus faibles ( 3 à $7 \mathrm{mg}$ ) que lorsque donnée par voie systémique, même s'il y a une absorption importante par les veines épidurales tôt après l'administration. ${ }^{11} \mathrm{La}$ position du cathéter le long de la colonne vertébrale n' affecte pas la production d'analgésie avec la morphine, ${ }^{12}$ même si le début de l'analgésie peut être retardé au niveau des segments éloignés du site de ponction. L'injection sous-arachnoïdienne exige de très petites doses $(0,2-0,5$ $\mathrm{mg}$ ), s'accompagne d'absorption systémique minimale et produit une analgésie prolongée, comme le fait l'administration épidurale (8-24 heures). ${ }^{13}$ Les opiacés solubles dans les lipides, dont les prototypes sont le fentanyl et le sufentanil, semblent agir différemment. Des doses se rapprochant des doses systémiques sont nécessaires pour obtenir l'analgésie et le cathéter doit être placé le plus près possible des segments dont dépend l'analgésie. La captation systémique est importante avec les opiacés lipophiliques et, si l'on utilise des infusions continue de fentanyl, les concentrations plasmatiques peuvent être identiques à celles trouvées lorsque la substance est administrée par voie intraveineuse pour obtenir des niveaux similaires d'analgésie. ${ }^{14,15}$ Cependant, si le cathéter est placé près du site chirurgical, des doses plus faibles de fentanyl sont requises avec obtention d'une concentration plasmatique subséquente plus faible. ${ }^{16}$ Les quantités plus importantes d'opiacés lipophiliques utilisés peuvent être mises en relation avec une captation systémique plus importante ainsi qu'avec une liaison non spécifique au niveau de la moelle. ${ }^{17}$ Les effets secondaires des opiacés "rachidiens » sont similaires à ceux rencontrés avec les voies alternatives d'administration et comprennent la dépression respiratoire, la rétention urinaire, du prurit, la nausée et les vomissements. La dépression opératoire après les opiacés rachidiens a été évaluée de façon beaucoup plus précise qu'avec n'importe quelle voie d'administration. ${ }^{18}$ Les facteurs de risque associés comprennent l'âge avancé, une fonction respiratoire compromise, et l'administration concommittente de sédatifs et d'opiacés par voie parentérale. Une dépression respiratoire précoce se produit à la suite de captation et redistribution systémique au centre respiratoire, et se produit jusqu'à 60 minutes après l'injection. ${ }^{18} \mathrm{La}$ dépression respiratoire tardive dépend des mouvements du liquide céphalo-rachidien dans la moelle ${ }^{19}$ et se produit plusieurs heures après l'administration (jusqu'à $18 \mathrm{~h}$ ). ${ }^{18}$ Avec la morphine, les risques de dépression respiratoire sont faibles mais imprévisibles. Le fentanyl semble présenter moins de risques de dépression respiratoire ${ }^{18}$ même si elle devient apparente avec des doses plus importantes, surtout en infusion épidurale.$^{20} \mathrm{La}$ dépression respiratoire peut être traitée avec la naloxone, alors que les autres effets secondaires peuvent être contrôlés avec des agents spécifiques (diphenhydramine, antiémétiques) ou la naloxone. Il n'y a pas de consensus quant aux sites et aux moyens de surveillance des patients qui reçoivent des opiacés rachidiens, même si la somnolence et la fréquence respiratoire peuvent être des instruments utiles de détection de la dépression respiratoire. De plus, des doses plus faibles et plus fréquentes de morphine peuvent diminuer les risques de dépression respiratoire. ${ }^{21}$ Des études de suivi commencent à paraître et indiquent que les opiacés rachidiens peuvent amener des bénéfices particuliers en termes de séjour hospitalier, morbidité, et mortalité. ${ }^{22,23}$

Opiacés et anesthésiques locaux par voie rachidienne Les efforts faits pour diminuer les doses et l'absorption d'opiacés ont conduit à l'utilisation de mélanges d'anesthésiques locaux et d'opiacés épiduraux. La combinaison de morphine $\left(0,1 \mathrm{mg} \cdot \mathrm{ml}^{-1}\right)$ et de bupivacaïne $0,1 \%$ s'est révélée être un mélange efficace, même si elle n'est pas meilleure que la morphine épidurale seule en termes d'analgésie, de besoins totaux d'opiacés ou d'incidence d'effets secondaires. ${ }^{24} \mathrm{De}$ façon similaire, les infusions de fentanyl épidural $\left(10 \mu \mathrm{g} \cdot \mathrm{ml}^{-1}\right)$ ou de sufentanil (1 $\mu \mathrm{g} \cdot \mathrm{ml}^{-1}$ ) additionnées à de la bupivacaïne $0,1 \%$ se sont révélés être efficaces pour l'analgésie postopératoire après des interventions variées. ${ }^{24}$ Cependant, lorsque des combinaisons similaires sont soumises à des évaluations rigoureuses avec attribution au hasard et double aveugle, on ne peut mettre en évidence de synergie des combinaisons lorsque comparées à l'opiacé seul. ${ }^{24}$ Des mélanges avec concentrations plus élevées de bupivacaïne $(0,5 \%)$ ont produit une analgésie profonde mais les patients ont aussi obtenu des niveaux d'analgésie thoracique élevés. ${ }^{25}$

\section{Opiacés transdermiques}

Le fentanyl pris par voie transdermique a fait l'objet de développements et d'études relativement importantes de son utilisation pour la douleur aiguë..$^{10,26} \mathrm{Le}$ développement d'un système thérapeutique transdermique et son application à l'utilisation de fentanyl procure un appareillage simple d'infusion continue non-invasive avec un niveau plasmatique thérapeutique d'une stabilité surprenante. ${ }^{26}$ Malheureusement, le système ne produit sa concentration thérapeutique qu'après 12 à 18 heures d'application, et le niveau plasmatique se maintient pour une plus longue période après le retrait. Ainsi, ce système peut produire une analgésie stable de façon prolongée, et des niveaux plasmatiques qui se maintiennent jusqu'à 72 heures après l'administration. Les doses disponibles sont de $25,50,75$ et $100 \mu \mathrm{g} \cdot \mathrm{h}^{-1}$. Le fentanyl transcutané peut amener une analgésie complète chez un petit nombre de patients à la suite de leur chirurgie mais, en général, demande des suppléments d'opiacés intraveineux ou 
intramusculaires en petites doses. ${ }^{10,27}$ L'incidence de nausées et vomissements a été élevé dans certaines études. ${ }^{10}$ De plus, certains sujets présentent une dépression respiratoire perceptible dans la plupart des études, ${ }^{10}$ surtout si les tampons de fentanyl sont utilisés. Les avantages des tampons de fentanyl transcutanés comprennent la facilité d'utilisation, une main d'oeuvre réduite, une réceptivité importante chez les patients et un niveau de base d'analgésie efficace qui demande une faible supplémentation en opiacés.

Les désavantages comprennent des doses prédéterminées non ajustables, un début lent, l'application de tampons en préopératoire, la nécessité d'administrer un supplément d'analgésie en salle de réveil, et des besoins de surveillance similaire par les infirmières comme pour tout autre système d'analgésie efficace.

\section{Autres agents administrés par voie rachidienne}

Les anesthésiques locaux ont été utilisés depuis longtemps par voie épidurale pour l'analgésie postopératoire. À la différence des agents opiacés, les anesthésisques locaux produisent de l'analgésie par bloc axonal, mais sont aussi capables de diminuer les réponses endocrines et métaboliques à la chirurgie, de réduire la neutropénie et la granulocytose, d'améliorer la balance azotée ${ }^{28}$ et peuvent aussi diminuer le risque d'incidents thrombo-emboliques. ${ }^{29} \mathrm{De}$ plus, la diminution de la dysfonction pulmonaire après la chirurgie thoracique et abdominale, et la récupération rapide de la motilité intestinale ont été mises en évidence. ${ }^{30}$ Les désavantages de l'anesthésie rachidienne par anesthésiques locaux comprenrient le bloc moteur, le bloc sympathique, la toxicité systémique et la rétention urinaire, tous des inconvénients qui peuvent être réduits en plaçant le cathéter près du site de chirurgie. La bupivacaïne, l'agent le plus couramment utilisé, est peu susceptible d'être toxique si les taux d'infusion se maintiennent en deçà de $30 \mathrm{mg}$ à l'heure. L'intérêt récent pour l'utilisation des cathéters rachidiens de calibre 28 pour infuser les anesthésiques locaux en analgésie postopératoire ${ }^{31}$ a été amoindri par la révélation que certains patients ont développé des syndromes de la queue de cheval associés au cathéter rachidien et aux anesthésiques locaux. ${ }^{32} \mathrm{La}$ clonidine, un agoniste alpha-2, s'est montrée peu efficace pour produire de l'analgésie lorsqu'utiliseé par voie épidurale. $^{33}$

\section{Blocs périphériques}

La transmission le long d'un nerf sensitif peut être interrompue par une variété de techniques incluant les anesthésiques locaux ou la cryoanalgésie. Un bloc efficace des nerfs périphériques par les anesthésiques locaux dans le but de produire une analgésie postopératoire peut être accompli par l'infiltration de la plaie, et des blocs de nerfs périphériques, intercostaux, de la plèvre et des blocs des plexus importants. ${ }^{34} \mathrm{~L}$ 'analgésie inter-pleurale procure un bloc partiel et continu de nombreux nerfs intercostaux et semble très efficace pour la douleur post-cholé cystectomi $^{35}$ mais semble être moins efficace après une thoracotomie. ${ }^{36}$ On n'a pas encore montré de bénéfice clair produit par l'utilisation de cette technique. ${ }^{37}$ Les complications comprennent le pneumothorax, l'effusion pleurale, la toxicité systémique par les anesthésiques locaux ainsi que des problèmes mécaniques. Les procédures intermittentes (bloc intercostaux, etc.) sont très efficaces mais les besoins d'administration répétée constituent un handicap pour l'administration à long terme. Pour cette raison, les blocs périphériques continus retiennent l'attention et ont été utilisés ạvec succès pour l'analgésie postopératoire. On a mis au point des techniques d'administration continue par cathéter pour les nerfs inter-costaux (mise en place au moment de la thoracotomie), le plexus brachial, le plexus lombaire ainsi que les sites sous-costaux ou paravertébraux. $^{34}$

La cryoanalgésie implique la congélation de nerfs exposés pendant la thoracotomie et peut amener une analgésie prolongée avec peu de complications. ${ }^{38}$

\section{Agents analgésiques non-opiacés}

L'utilisation d'agents anti-inflammatoires dans la période postopératoire augmente puisque des travaux mentionnent que des agents injectables tel le kétorolac ont des propriétés analgésiques intéressantes surtout pour la douleur faible à modérée. ${ }^{39}$ Lorsqu'il y a douleur sévère, ces agents peuvent réduire l'utilisation d'opiacés. On peut mentionner parmi les autres agents anti-inflammatoires non stéroïdiens le piroxicam, le diclofenac et l'indométhacine, ${ }^{40}$ et tous peuvent être administrés par voie orale ou rectale. Leurs effets secondaires concernent surtout le tractus gastrointestinal, la fonction plaquettaire et le saignement possible

\section{Conclusion}

De nombreuses approches d'analgésie postopératoire continuent de se développer à mesure que l'intérêt pour le contrôle de la douleur aiguë augmente, surtout dans le domaine de l'anesthésie. Les progrès ultérieurs apparaîtront dans le futur surtout dans le domaine de l'analgésie préventive et la réduction de la douleur postopératoire par contrôle de la plasticité de la moelle. ${ }^{41,42}$ Le concept d'analgésie multi-modale ou balancée ${ }^{43}$ dans laquelle on utilise plusieurs agents spécifiques pour contrôler des voies spécifiques de conduction de la douleur est un autre secteur qui pourra amener des progrès dans l'analgésie postopératoire.

\section{Références}

(Voir page R55) 